\title{
Síndrome por infusión de propofol: reporte de un caso
}

\author{
Viviana Martínez ${ }^{*}$, Martín Angulo†, Marcelo Barbato‡
}

\section{Resumen}

El propofol es un anestésico ampliamente utilizado en las unidades de cuidados intensivos. Su empleo puede provocar un efecto adverso poco frecuente pero extremadamente grave, conocido como síndrome por infusión de propofol (SIP), caracterizado por inestabilidad hemodinámica, rabdomiólisis, acidosis láctica y disfunción multiorgánica. Presentamos un caso de SIP con desenlace fatal, discutiendo su fisiopatología, clínica y consideraciones a tener en cuenta al emplear propofol.

Palabras clave: SIINDROME DE INFUSIÓN DE PROPOFOL

\section{Key words: $\quad$ PROPOFOL INFUSION SYNDROME}

\section{Introducción}

El síndrome por infusión de propofol (SIP) es un cuadro infrecuente pero extremadamente grave secundario a la administración de propofol, fármaco ampliamente utilizado en las unidades de cuidados intensivos (UCI) ${ }^{(1)}$. La inestabilidad hemodinámica con acidosis láctica, rabdomiólisis y disfunción multiorgánica son las manifestaciones preponderantes, determinando en muchos casos la muerte del paciente. Si bien el principal factor de riesgo para su desarrollo consiste en la administración prolongada de dosis elevadas de propofol, se han des- crito casos en pacientes que recibieron dosis relativamente bajas por breves períodos ${ }^{(2,3)}$. Esto remarca la necesidad de mantener un elevado índice de sospecha y realizar un estricto seguimiento de aquellos pacientes tratados con este fármaco.

\section{Caso clínico}

Presentamos el caso de un hombre de 25 años que sufrió un traumatismo encefalocraneano (TEC) grave, ingresando a la UCI en coma, estable hemodinámicamente y con buen intercambio gaseoso. La tomografía axial computarizada evidenció una contusión hemorrágica temporal y un hematoma subdural fronto-témporo-parietal derechos con efecto de masa, que fueron evacuados mediante neurocirugía de urgencia. En el posoperatorio presentó hipertensión intracraneana (HIC) pese a las medidas terapéuticas iniciales (sedoanalgesia, bloqueo neuromuscular, osmoterapia, hiperventilación). Dada la persistencia de HIC al tercer día de evolución se inició infusión de propofol (dosis 2,5 a 3,3 mg/kg/h) y se realizó craniectomía descompresiva. Mantuvo HIC refractaria, por lo que al sexto día se colocó un drenaje lumbar para extracción de líquido cefalorraquídeo, logrando controlar la misma. Se comenzó el retiro gradual de las medidas instauradas, suspendiéndose la infusión de propofol al séptimo día del ingreso (quinto día de infusión).

Concomitantemente con la mejoría de la HIC el paciente presentó un deterioro del intercambio gaseoso, fiebre e hipotensión arterial. Se constataron consolida-

\footnotetext{
* Médico intensivista, Centro de Tratamiento Intensivo, Hospital Maciel, Administración de los Servicios de Salud del Estado. Montevideo, Uruguay.

† Médico intensivista, Centro de Tratamiento Intensivo, Hospital Maciel, Administración de los Servicios de Salud del Estado. Montevideo, Uruguay.

Prof. Adj. Fisiopatología, Facultad de Medicina, Universidad de la República. Montevideo, Uruguay.

Asistente de Medicina Intensiva, Facultad de Medicina, Universidad de la República. Montevideo, Uruguay.

$\ddagger$ Médico intensivista, Jefe del Centro de Tratamiento Intensivo, Hospital Maciel, Administración de los Servicios de Salud del Estado. Montevideo, Uruguay.

Departamento de Fisiopatología. Hospital de Clínicas, Av. Italia s/n, piso 15. Montevideo, Uruguay

Los autores declaran no tener conflicto de intereses.

Correspondencia: Dr. Martín Angulo. Correo electrónico: martin.angulo@hc.edu.uy

Recibido: $24 / 4 / 17$

Aprobado: $14 / 8 / 17$
} 
ciones pulmonares bilaterales y desarrolló Haemophilus influenzae en cultivo de secreciones traqueales, recibiendo tratamiento específico con ampicilina-sulbactam sin mejoría. En las siguientes 24 horas el paciente desarrolló un estado de shock refractario, sin respuesta a la reposición con cristaloides, apoyo con vasopresores a dosis elevadas y administración de corticoides (hidrocortisona $300 \mathrm{mg} / \mathrm{día}$ ). No se constataron arritmias cardíacas. Pese a que el shock refractario se instaló en las 24 horas siguientes a la suspensión del propofol, debido a la elevada dosis acumulada recibida se planteó un SIP. El diagnóstico fue apoyado por la presencia de acidosis metabólica con hiperlactatemia $(10 \mathrm{mEq} / \mathrm{L})$, rabdomiólisis (creatina fosfoquinasa [CPK] $76.000 \mathrm{UI} / \mathrm{L}$ ) e hipertrigliceridemia $(354 \mathrm{mg} / \mathrm{dL})$. Se comenzó tratamiento con gluconato de calcio e infusión de soluciones glucosadas. Instaló injuria renal aguda oligoanúrica, hiperpotasemia, falla hepática y trastorno de la coagulación. Se realizó hemodiálisis extendida, sin mejoría. Falleció al décimo día del ingreso.

\section{Discusión}

El SIP constituye un cuadro poco frecuente pero extremadamente grave, caracterizado por shock y disfunción orgánica múltiple secundaria a la infusión del fárma$\mathrm{co}^{(1)}$. Su incidencia real es desconocida, ya que la mayoría de los datos provienen de reportes de casos, sumado al hecho de no contar con criterios unificados para su diagnóstico. Cremer y colaboradores reportaron una incidencia de $10 \%$ en adultos neurocríticos tratados con propofol, pero esta aumentaba a $31 \%$ en aquellos pacientes que recibieron dosis mayores a $6 \mathrm{mg} / \mathrm{kg} / \mathrm{h}^{(4)}$. Si bien un estudio prospectivo más reciente encontró una incidencia mucho menor $(1,1 \%)$, los pacientes incluidos recibieron dosis más bajas de propofol ${ }^{(5)}$. Los reportes de mortalidad atribuible al SIP oscilan entre 30\% y $80 \%{ }^{(5,6)}$. La dosis acumulada de propofol, la presencia de síntomas cardíacos, hipotensión arterial, fiebre, acidosis metabólica, falla renal, la edad avanzada y el TEC son factores de riesgo que aumentan la mortalidad del SIP $^{(2,6)}$. El paciente presentaba numerosos factores de riesgo para el desarrollo de un SIP grave (elevada dosis acumulada del fármaco, TEC, shock, fiebre, falla renal, acidosis metabólica).

La fisiopatología del SIP es en gran medida incierta y probablemente multifactorial. El principal responsable de la lesión y muerte celular parece ser el desequilibrio entre oferta y demanda energética ${ }^{(7)}$. El propofol interfiere con la fosforilación oxidativa provocando el desacople de la cadena respiratoria mitocondrial e impide la betaoxidación de ácidos grasos por inhibición de la actividad de la carnitina palmitoil-transferasa $\mathrm{I}^{(7)}$. Esto determina un estado de penuria energética celular y pro- mueve la acumulación de ácidos grasos libres en distintos órganos. El déficit energético afecta especialmente a las células musculares esqueléticas y cardíacas, provocando lisis celular y desarrollo de rabdomiólisis y disfunción miocárdica, respectivamente ${ }^{(8)}$. La rabdomiólisis conlleva a la liberación de productos intracelulares como mioglobina, $\mathrm{CPK}$, potasio y ácido láctico, pudiendo generar o agravar una insuficiencia renal. El propofol inhibe los receptores betaadrenérgicos y bloquea los canales de calcio cardíacos, pudiendo favorecer el desarrollo de las manifestaciones cardiovasculares ${ }^{(9)}$. Otros factores que pueden contribuir al desarrollo de SIP incluyen la disminución de las reservas de carbohidratos y el estrés propio de la enfermedad crítica. Los pacientes neurocríticos parecen ser especialmente susceptibles al desarrollo de formas letales de SIP, lo que podría estar vinculado a la gran descarga endógena y administración exógena de catecolaminas en este tipo de pacientes ${ }^{(2,7)}$.

Las dosis elevadas de propofol (tasa de infusión y duración de la misma) constituyen el principal factor de riesgo para el desarrollo del SIP y aumentan la mortalidad del mismo ${ }^{(2,4)}$. Esto ha llevado a distintos autores a recomendar que la dosis de propofol no supere los 4 $\mathrm{mg} / \mathrm{kg} / \mathrm{h}$, por un período máximo de 48 horas $^{(8-10)}$. No obstante, el SIP puede desarrollarse tras la administración de dosis menores durante períodos de tiempo más $\operatorname{cortos}^{(2)}$. En nuestro paciente, si bien la tasa de infusión se mantuvo siempre por debajo de $4 \mathrm{mg} / \mathrm{kg} / \mathrm{h}$, la misma se extendió por un período de cinco días. Esto se debió a que se priorizó el tratamiento de la HIC severa y extremadamente refractaria. Tras cuatro días de infusión, la presión intracraneana logró controlarse mediante la colocación de un drenaje lumbar, permitiendo el descenso y suspensión de la infusión de propofol al día siguiente. Otros posibles factores de riesgo para el desarrollo de SIP son la existencia de patologías graves (especialmente injuria neurológica), el uso de vasopresores o glucocorticoides, el déficit de hidratos de carbono y la presencia de cardiopatía previa ${ }^{(10)}$. A su vez, la existencia de alteraciones mitocondriales subyacentes representaría un riesgo aumentado para el desarrollo de $\operatorname{SIP}^{(10)}$.

En nuestro paciente los primeros signos de deterioro sistémico consistieron en fiebre, hipoxemia e hipotensión arterial, los cuales fueron atribuidos al desarrollo de una neumonía asociada a la ventilación mecánica (consolidaciones pulmonares y aislamiento de $H$. influenzae en secreciones traqueales). Pese al tratamiento antibiótico específico el paciente presentó un marcado deterioro clínico (shock refractario, acidosis metabólica, injuria renal), obligando a la búsqueda de diagnósticos diferenciales y al planteo de SIP. Como ya se detalló anteriormente, no existe consenso acerca de los criterios diagnósticos del SIP. El cuadro se caracteriza por disfunción 
hemodinámica y shock rápidamente progresivo, rabdomiólisis y acidosis láctica, asociando injuria renal aguda e hiperpotasemia ${ }^{(7)}$. Son frecuentes las arritmias cardíacas, principalmente la bradicardia, y alteraciones electrocardiográficas similares a las observadas en el síndrome de Brugada (elevación del segmento ST en V1-V3) ${ }^{(10)}$. Suele cursar con hipertrigliceridemia e insuficiencia hepatocítica.

No existe un tratamiento específico para el SIP establecido. La primera medida consiste en suspender inmediatamente la administración del fármaco. Las medidas de sostén hemodinámico incluyen el apoyo vasopresor con catecolaminas, pudiendo requerir la utilización de un marcapaso externo en el caso de bradiarritmias refractarias ${ }^{(10)}$. Se ha sugerido que en los cuadros más graves debería considerarse el uso de milrinona, glucagón o calcio, así como la utilización de oxigenación por membrana extracorpórea ${ }^{(9)}$. La injuria renal y la hiperpotasemia suelen requerir la implementación de tratamiento sustitutivo de la función renal. Además, se ha planteado que tanto la hemodiálisis como la hemofiltración podrían colaborar con la eliminación del propofol y sus metabolitos ${ }^{(10)}$. Se recomienda mantener un aporte de carbohidratos de entre 6-8 mg/ $\mathrm{kg} / \mathrm{min}$. En el caso presentado, además de las medidas de sostén hemodinámico y respiratorio, se realizó tratamiento sustitutivo de la función renal mediante hemodiálisis, aporte de gluconato de calcio y carbohidratos, sin lograr estabilización ni mejoría del cuadro.

En suma, el SIP es un cuadro relativamente poco frecuente pero potencialmente letal. Si bien el riesgo se incrementa a mayor velocidad de infusión y duración del tratamiento, el cuadro puede desarrollarse con infusiones cortas a dosis menores de $4 \mathrm{mg} / \mathrm{kg} / \mathrm{h}$. Se debe mantener un alto índice de sospecha en todo paciente tratado con propofol, monitoreando en forma diaria elementos que sugieran su aparición (electrocardiograma, determinación sanguínea de lactato, CPK, triglicéridos, etcétera).

\section{Abstract}

Propofol is a widely used anesthetic in the Intensive Care Unit. At times it may cause rather an unusual adverse reaction known as propofol infusion syndrome (PRIS), characterized by hemodynamic instability, rhabdomyolysis, lactic acidosis, and multiple-organ dysfunction. The study presents a case of PRIS with fatal outcome, including a discussion of its physiopatho- logy, clinical aspects and considerations to be borne in mind when using propofol.

\section{Resumo}

O propofol é um anestésico amplamente utilizado nas Unidades de Cuidados Intensivos. Sua utilização pode provocar um efeito adverso pouco frequente mas extremamente grave conhecido como síndrome por infusão de propofol (SIP), caracterizado por instabilidade hemodinâmica, rabdomiólise, acidose lática e disfunção múltipla de órgãos. Apresentamos um caso fatal de SIP e discutimos a fisiopatologia, clínica e os aspectos que devem ser considerados quando se utiliza esta droga.

\section{Bibliografía}

1. Fudickar A, Bein B, Tonner PH. Propofol infusion syndrome in anaesthesia and intensive care medicine. Curr Opin Anaesthesiol 2006; 19(4):404-10.

2. Krajèová A, Waldauf $\mathbf{P}$, Andì $\mathbf{M}$, Duška F. Propofol infusion syndrome: a structured review of experimental studies and 153 published case reports. Crit Care 2015; 19:398.

3. Merz TM, Regli B, Rothen HU, Felleiter P. Propofol infusion syndrome: a fatal case at a low infusion rate. Anesth Analg 2006; 103(4):1050.

4. Cremer OL, Moons KG, Bouman EA, Kruijswijk JE, de Smet AM, Kalkman CJ. Long-term propofol infusion and cardiac failure in adult head-injured patients. Lancet 2001; 357(9250):117-8.

5. Roberts RJ, Barletta JF, Fong JJ, Schumaker G, Kuper PJ, Papadopoulos S, et al. Incidence of propofol-related infusion syndrome in critically ill adults: a prospective, multicenter study. Crit Care 2009; 13(5):R169.

6. Fong JJ, Sylvia L, Ruthazer R, Schumaker G, Kcomt M, Devlin JW. Predictors of mortality in patients with suspected propofol infusion syndrome. Crit Care Med 2008; 36(8):2281-7.

7. Vasile B, Rasulo F, Candiani A, Latronico N. The pathophysiology of propofol infusion syndrome: a simple name for a complex syndrome. Intensive Care Med 2003; 29(9):141725.

8. Fudickar A, Bein B. Propofol infusion syndrome: update of clinical manifestation and pathophysiology. Minerva Anestesiol 2009; 75(5):339-44.

9. Mirrakhimov AE, Voore P, Halytskyy O, Khan M, Ali AM. Propofol infusión syndrome in adults: a clinical update. Crit Care Res Pract 2015; 2015:260385.

10. Otterspoor LC, Kalkman CJ, Cremer OL. Update on the propofol infusion syndrome in ICU management of patients with head injury. Curr Opin Anaesthesiol 2008; 21(5):54451. 